\title{
Patient, staff, and clinician perspectives on implementing electronic communications in an interdisciplinary rural family health practice
}

\author{
Feng Chang ${ }^{1}$, Thivaher Paramsothy ${ }^{2}$, Matthew Roche ${ }^{2}$ and Nishi S. Gupta ${ }^{1}$ \\ ${ }^{1}$ School of Pharmacy, University of Waterloo, Waterloo, Ontario, Canada \\ ${ }^{2}$ School of Public Health and Health Systems, University of Waterloo, Waterloo, Ontario, Canada
}

\begin{abstract}
Aim: To conduct an environmental scan of a rural primary care clinic to assess the feasibility of implementing an e-communications system between patients and clinic staff. Background: Increasing demands on healthcare require greater efficiencies in communications and services, particularly in rural areas. E-communications may improve clinic efficiency and delivery of healthcare but raises concerns about patient privacy and data security. Methods: We conducted an environmental scan at one family health team clinic, a high-volume interdisciplinary primary care practice in rural southwestern Ontario, Canada, to determine the feasibility of implementing an e-communications system between its patients and staff. A total of 28 qualitative interviews were conducted (with six physicians, four phone nurses, four physicians' nurses, five receptionists, one business office attendant, five patients, and three pharmacists who provide care to the clinic's patients) along with quantitative surveys of 131 clinic patients. Findings: Patients reported using the internet regularly for multiple purposes. Patients indicated they would use email to communicate with their family doctor for prescription refills (65\% of respondents), appointment booking $(63 \%)$, obtaining lab results $(60 \%)$, and education (50\%). Clinic staff expressed concerns about patient confidentiality and data security, the timeliness, complexity and responsibility of responses, and increased workload. Conclusion: Clinic staff members are willing to use an e-communications system but clear guidelines are needed for successful adoption and to maintain privacy of patient health data. E-communications might improve access to and quality of care in rural primary care practices.
\end{abstract}

Key words: electronic communications; family practice; outpatient health services; patient survey; qualitative interview; rural primary care

Received 11 July 2016; revised 14 September 2016; accepted 14 September 2016;

first published online 20 December 2016

\section{Background}

Canadian policymakers have struggled to identify optimal approaches to providing timely and quality healthcare to all Canadians. This is particularly a challenge for those who live in rural regions, as they

Correspondence to: Feng Chang, BScPhm, PharmD, Chair of Rural Pharmacy, Gateway Centre of Excellence in Rural Health, Assistant Professor, School of Pharmacy, University of Waterloo, 10A Victoria Street South, Kitchener, Ontario, Canada N2G1C5. Email: feng.chang@uwaterloo.ca

(C) Cambridge University Press 2016 are more likely to have unmet healthcare needs, particularly access to specialist physician services, than those in urban areas (Sibley and Weiner, 2011).

An estimated $16.5 \%$ of Canadians are 65 years of age or older in 2016 , a percentage projected to increase to $23 \%$ by 2031 (Statistics Canada, 2015). As people age they tend to develop chronic conditions, so this increasing proportion of older Canadians with growing medical needs is expected to strain healthcare resources. In addition, the increasing complexity of healthcare and rising demand for chronic disease management services 
have required more physician time than is often available (Virji et al., 2006). Efficient communications between primary care providers and patients is one mechanism by which quality of and access to care could be improved. Indeed, the 2012 Physician Services Agreement between the Ontario Medical Association and the province's Ministry of Health and Long-Term Care stressed the importance of modernising primary care delivery and reducing wait times specifically through e-communications that allow patients to interact with their doctors more readily (Ontario Ministry of Health and Long-Term Care, 2013).

Doctor-patient e-communications has been examined in multiple studies (Couchman et al., 2001; Baker et al., 2005; Leong et al., 2005; Liederman et al., 2005; Virji et al., 2006; Kummervold and Johnsen, 2011; Bishop et al., 2013; Keplinger et al., 2013; Crotty et al., 2014; de Jong et al., 2014; Garrido et al., 2014; Plener et al., 2014; Newhouse et al., 2015). Most studies have found that e-communications with patients has been positive, and several have found that e-communications can also improve clinic efficiency and cost-effectiveness (Baker et al., 2005; Liederman et al., 2005; Virji et al., 2006; Bishop et al., 2013; Keplinger et al., 2013; Crotty et al., 2014; de Jong et al., 2014; Plener et al., 2014). Some researchers have also found improved treatment adherence and health outcomes after implementing e-communications (de Jong et al., 2014; Garrido et al., 2014; Plener et al., 2014). However, information about the feasibility of implementing e-communications between patients and healthcare providers in rural regions is lacking.

The purpose of this study was to perform an environmental scan of a rural primary care clinic to assess the feasibility of adopting an e-communications system to improve communication between patients and staff.

\section{Methods}

The study received ethics approval from the University of Waterloo's Office of Research Ethics. This study surveyed 131 patients at a family health team clinic, a high-volume medical practice in rural southwestern Ontario (population 6867; Canada 2011 Census) with 10 physicians, 11 nurses, three nurse practitioners, and other allied health professionals. This quantitative survey assessed the technological capabilities of the clinic's patients and their attitudes towards technology (see Appendix). Over a six-week period, patients were invited to complete the survey by the clinic's receptionists at the time of the patients' appointments; however, during busy times the receptionists may have chosen to not disrupt operations by asking patients to complete the survey. Surveys were completed in the waiting room and submitted before leaving the clinic. Patients who completed the survey were invited to participate in qualitative interviews to provide additional depth to their responses. Survey data were entered into a Microsoft Excel spreadsheet and were used to calculate frequencies and percentages.

During a clinic meeting, the study's authors informed clinic staff and physicians about the study's purpose. Staff and physicians were then invited to participate in a half-hour semi-structured interview conducted by one of two student researchers in a private room at the clinic. In addition, all three pharmacies in the township were contacted by phone and invited to participate in the study. Three pharmacists were interviewed, one from each pharmacy, by the same student researchers (see Appendix for the qualitative survey questions). All interviews were recorded and transcribed. Transcripts were verified by a second investigator and analysed using thematic coding.

\section{Results}

\section{Patient characteristics}

Of the 131 patients surveyed $70 \%$ were female. The mean age of respondents was 45 years, with ages ranging from 17 to 92 . Half of respondents $(50.4 \%)$ lived in Listowel, Ontario. Half (49.6\%) had educational training beyond secondary school/ high school and half (49.6\%) had completed that level of education or less. Using a five-point Likert scale ranging from excellent to poor, the average self-reported health status was 3.47 , that is, between good and very good health (see Table 1).

\section{Current use of technology}

A total of $80 \%$ of respondents indicated they had access to the internet at home, and $49.6 \%$ indicated they had access in other locations such as 
Table 1 Patient characteristics

\begin{tabular}{lc}
\hline Characteristic & Surveyed patient sample $(n=131)$ \\
\hline Age & \\
$\quad$ Mean years & 45 \\
Range in years $[n(\%)]$ & $17-92$ \\
$<65$ years $[n(\%)]$ & $112(85.5)$ \\
$\geqslant 65$ years $[n(\%)]$ & $19(14.5)$ \\
Sex $[n(\%)]$ & \\
Female & $92(70.2)$ \\
Male & $39(29.8)$ \\
Geographical location $[n(\%)]$ & $4(3.0)$ \\
$\quad$ Kitchener-Waterlo & $66(50.4)$ \\
Listowel & $58(44.3)$ \\
Other & $3(2.3)$ \\
Did not answer & \\
Education level $[n(\%)]$ & $65(49.6)$ \\
Secondary school/high school or less & $65(49.6)$ \\
Training beyond secondary school/high school & $1(0.8)$ \\
Did not answer & \\
Self-reported health status (five-point scale) & $3.47(\mathrm{good}$ to very good) \\
$\quad$ Average rating [no. (interpretation)] &
\end{tabular}

at work, libraries and via mobile phones. Of those surveyed, $86 \%$ had an email account and $70 \%$ spent more than an hour a day on average checking email. The most common internet uses were conducting searches $(79 \%)$, reading and replying to email (62\%), using social media $(55 \%)$ and obtaining news, weather or sports information $(50 \%)$. Patients also indicated they use the internet to learn about health and medical issues, with $81 \%$ indicating they had used it for either purpose at some point.

\section{Attitudes regarding communication}

Patients were asked to indicate their likelihood of using email to communicate with family health team staff on a scale from zero to 10 (zero being not likely at all and 10 being very likely). About $28 \%$ rated their likelihood between zero and four, $12 \%$ rated their likelihood as five, and $60 \%$ rated their likelihood as six or greater. Almost half $(48 \%)$ of patients indicated they would like to use email to communicate with their family doctor, $28 \%$ indicated they would not like to use email for this purpose, and $21 \%$ were unsure. Patients indicated getting prescription refills $(65 \%)$, booking appointments $(63 \%)$, obtaining lab test results $(60 \%)$, and furthering their education $(50 \%)$ as potential uses for email communications.

\section{Perspectives of patients and clinic staff}

Except for one patient and one nurse, all participants indicated that they use the internet to some extent, mostly for personal communication. Only the physicians and a few of the nurses indicated that they use email professionally for tasks such as scheduling meetings, communicating with individuals at other workplaces, and professional development. None of the phone nurses or office staff had an email address associated with the clinic.

The clinic's chain and methods of communications appeared to be sources of frustration for most clinic staff. Matters involving patient interaction accounted for a smaller portion of challenges identified by clinic staff. Most issues with patients were attributable to available clinic appointments not meeting patient demand. An inability to communicate with patients who are unable to contact the clinic during the day and timeliness of responses to patient inquiries were identified as obstacles that are likely attributable to internal communications processes.

A lack of dialogue relative to phone conversations, increased staff workload and timeliness of email replies were identified as main concerns. Interviewees indicated that email might go unnoticed if the intended recipient was out of the office, if the message was not seen because it was diverted to the junk email folder, or if the email did not reach its intended recipient. 
Most clinic staff indicated that an appropriate response time to email was within seven days of having received it, but a few indicated that one to two days were appropriate. However, patients indicated that their ideal response time was between $30 \mathrm{~min}$ to two days, although they would expect a reply in one to two days, with one person indicating seven days. Patients indicated they would follow-up e-communications with phone calls or additional email if they were not replied to within a period they felt was appropriate. Some medical staff thought patients may expect email to be a quicker way to communicate with clinical staff and may rely on having issues resolved by email rather than by an office visit. Most staff members felt that patients should not initiate e-communications. Physicians expressed concern over the potential for abuse of e-communications by patients they find challenging to manage by phone.

Both clinic staff and patients were concerned about maintaining data privacy and confidentiality, the main concern being security of patient health information. Staff members also indicated that system-use guidelines would need to be clear and well defined and that patients should be required to sign a consent indicating they understand how the system works and how their personal health information will be used. Staff members also recommended training patients to realise the full potential of the system.

Several staff members expected they could respond to email messages whenever they had spare time, rather than having to make time to reply. Staff also thought that e-communications would be more successful in reaching patients than would messages left on answering machines.

When asked which activities are best suited to e-communications, the most common responses were scheduling, prescription renewals, general inquiries, and obtaining test results. When asked which activities would be inappropriate for e-communications, the most common responses were replying to urgent matters and conveying positive diagnostic test results.

Some suggested that scheduling and medical inquiries should be separate e-communications functions. A less popular suggestion was to send all inquiries, both scheduling and medical, to receptionists, who would then forward them to appropriate clinic personnel.

Most interviewees noted that older patients might be unreceptive or unable to use email and, conversely, that younger patients might be more eager to have an e-communications system implemented. Some were concerned that the Mennonite patients in this practice, a Christian group in the region that discourages use of technology, might be reluctant to use e-communications. Clinic staff were asked to rate their readiness and willingness to use e-communications on a scale from zero to 10 (zero representing they were not ready/willing at all, and 10 representing they would be ready/willing to use email the next day). The average rating by clinic staff (readiness -4.83 , willingness -4.88 ) and by patients (readiness -4.57 , willingness -5.24 ) were similar. Most staff compared the implementation of an e-communications system to a previous update to the clinic's computer system, although one receptionist noted that staff attitudes on the system update were split, potentially posing a barrier to adopting an e-communications system.

\section{Local pharmacists}

Three pharmacists, one from each of the three community pharmacies in the region, were interviewed to obtain their opinions on implementing an e-communication system. All communicate from five to 20 times a day with the family health team clinic, mainly to clarify concerns about prescriptions, answer questions, obtain authorisations and make recommendations. Communication is primarily by fax, with occasional phone calls for follow-up and clarification.

Pharmacists were also concerned about maintaining patient privacy and confidentiality, but were not concerned about response timeliness. Two pharmacists indicated that implementing an e-communications system would require significant workflow changes to be successful. All three were concerned about filling prescriptions over the internet because the security and authenticity of the connection might not meet legal requirements of the Ontario College of Pharmacists, the province's registering and regulating body for pharmacy practice. In spite of these concerns, all pharmacists indicated they would be willing to communicate with clinic staff by email.

\section{Discussion}

We surveyed patients as well as interviewed patients, staff and health-care providers at a rural primary care clinic to better understand barriers and 
facilitators to implementing an e-communications system. This rural Ontario clinic has a large patient call volume, which has overwhelmed staff. Improving patient-clinic communications may increase efficiency, productivity and delivery of healthcare.

Patients at this rural clinic are generally experienced at and comfortable with using email. About $86 \%$ have an email account and about $70 \%$ spend on average more than an hour a day checking email. Reading and replying to email is a main internet use, second only to conducting searches.

E-communications between patients and clinic staff may offer additional ways for patients to acquire information and become more involved in their health and healthcare. Several studies have demonstrated improved patient involvement as a result of e-communications (de Jong et al., 2014; Garrido et al., 2014; Plener et al., 2014; Newhouse et al., 2015). Similar to findings of other researchers (Couchman et al., 2001; Virji et al., 2006; Singh et al., 2009; Lam et al., 2013; Schickedanz et al., 2013; Crotty et al., 2014), the rural patients in our study also indicated a willingness to use e-communications with physicians and clinic staff.

Our findings are consistent with those of earlier studies that examined patient preference for email use, particularly the desire to use email to obtain prescription refills and obtain lab test results (Virji et al., 2006; Singh et al., 2009; Ye et al., 2010; Lam et al., 2013; Schickedanz et al., 2013; de Jong et al., 2014). Almost half of patients (48\%) expressed a desire to use email to communicate with physicians. However, as email is an unstructured form of communication - that is, a medium without standardised input fields - messages from patients might be difficult to understand or interpret. Standardised or closed data fields might help patients to enter crucial health information so physicians have sufficient and appropriate information to respond. Such portals have been implemented successfully in one study (Crotty et al., 2014).

Both clinic staff and patients expressed concern about security and privacy of e-communications. Security is essential if personal health data are transmitted. Santana et al. (2011) examined e-communications between doctors and patients in Europe and the solutions adopted to mitigate concerns about using online systems. Danish patients have two ways of communicating with a doctor: using Denmark's official eHealth portal (www.sundhed.dk), which requires a specific digital signature, and through their doctor's personal website, which requires a secure login. For both methods, e-communications occurs through an encrypted web interface (Santana et al., 2011). A similar system could be adopted by the family health team clinic to ensure that personal health information is transmitted securely.

Staff were concerned that implementing an e-communication system could increase their workload. However, Kittler et al. (2004) provide evidence that this may not be so. Staff members at 10 primary care clinics in Boston, Massachusetts were surveyed before and after implementing the Patient Gateway, a system that provides secure electronic communications between patients and clinic staff. Initially, 58\% of respondents thought the new system would increase their workload, $10 \%$ thought it would decrease it, and $25 \%$ were unsure. The post-implementation survey revealed that $63 \%$ of respondents found that their workload did not change after introducing the Patient Gateway (Kittler et al., 2004). A smaller, more recent study examined physicians' attitudes about implementing a secure patient communications system and found that, before implementation, $64 \%$ felt it would increase their workload. Post-implementation, $13 \%$ of physicians thought their workload had increased (Keplinger et al., 2013). Other studies have found that increase in workload with e-communications adoption is modest and may be offset by reduced clinic visits (Kummervold and Johnsen, 2011; Garrido et al., 2014).

Kummervold and Johnsen (2011) analysed the length of time it took physicians to respond to patients over the internet, as well as the correlation between the length of patient questions and subsequent physician responses. Using information from 14 physicians in seven offices, and 1113 messages, they found that physicians spent $2.3 \mathrm{~min}$ on average answering patient inquiries. The shortest $25 \%$ of messages required $2.1 \mathrm{~min}$ on average to answer and the longest $25 \%$ took $2.4 \mathrm{~min}$ on average to answer. Even questions three times longer than average required only $18 \%$ more time for a physician to response (Kummervold and Johnsen, 2011).

Crotty et al. (2014) found substantial variability in email volume for physicians within the same practice. Using the same messaging service, the interquartile range of patient-to-physician messages per physician per year was from 55 to 521 . These findings suggest that changes in physician workload 
associated with implementing e-communications are complex, and each physician's message volume can vary greatly even within the same clinic. The renegotiation of daily schedules and time for e-consultation may be necessary - the latter being a method already employed by some physicians (Bishop et al., 2013) - to minimise demand on physicians' time.

The delay in responding makes e-communications unsuitable for urgent matters, and discussion of positive diagnostic test results generally necessitates a face-to-face conversation between doctors and patients. The timeliness of email replies and determining which matters are considered urgent as reported by the family health team staff have been issues at other clinics. Sibley and Weiner's study (2011) on smart phone use for clinic communications found that physician and nurse perceptions of urgency differed. Often, physicians who did not perceive an issue to be urgent did not respond to an email despite a response request from a nurse. A possible solution could be deciding which patient or health issues should be dealt with urgently. Nonetheless, Sibley and Weiner (2011) reported a perceived improvement in efficiency with e-communications relative to pagers, the clinic's previous communications method.

Staff attitudes towards e-communications were divided and willingness to use such a system was reluctant, which could pose barriers to the family health team adopting an e-communications system. One explanation may be the perceived increase in workload for staff, accompanied by significant workflow and operations modifications, whereas benefits, particularly financial, were perceived by staff to be negligible. Reimbursement has been proposed as a possible mechanism to overcome barriers to adopting e-communications (Patt et al., 2003; Bishop et al., 2013; Crotty et al., 2014). Reimbursing physicians for chronic disease management by e-communications could promote physician-patient communications (Hobbs et al., 2003).

\section{Limitations}

Patients who chose to complete the quantitative survey may have differed from the clinic's patients overall, thus introducing self-selection bias. The proportion of older patients and the distribution by gender in our study may not reflect that of the clinic's population. Moreover, we do not have data on the survey response rate - that is, the number of patients who were asked by receptionists to

Primary Health Care Research \& Development 2017; 18: 149-160 complete the questionnaire but had declined to do so. Although the receptionists asked patients to complete the survey when they arrived for their appointments, when the office was busy or running behind schedule they may not have asked patients to participate. This latter source of bias may have been more a function of clinic workload than of patient characteristics, but it is a type of selection bias that may have influenced our findings.

\section{Conclusion}

We evaluated the feasibility of implementing an e-communications system between patients and staff at a rural primary care clinic. Overall, patients reported they were willing to communicate electronically with their physicians. Clinicians and staff, however, had reservations about e-communications. If the clinic adopted such a system, clinicians and staff felt that clear guidelines and processes would be necessary, along with measures to ensure patient privacy and health data security. Introducing e-communications might improve access to care in this rural primary care clinic, but it is unclear how patient health outcomes or clinic costs might be affected. Further research at this clinic may reveal whether e-communications improves patient health outcomes and delivery of healthcare.

\section{Acknowledgements}

The authors thank the physicians, staff and administrators at the family health team clinic for their participation and kind assistance with coordinating study activities; Jenny Seguin and Maral Mirhosseini, University of Waterloo School of Pharmacy students, for their help with data collection and transcription; Dr. Claudio Munoz, Gateway Centre of Excellence in Rural Health, for generous input and guidance; and Joe Petrik for editing and formatting the manuscript.

\section{Financial Support}

This research did not receive funding from any agency, commercial or not-for profit sector.

\section{Conflicts of Interest}

The authors report no conflicts of interest. 


\section{Ethical Standards}

The authors assert that all procedures contributing to this work comply with the ethical standards of the relevant national and institutional guidelines on human experimentation adhered to and enforced by the University of Waterloo's Office of Research Ethics and with those of the Helsinki Declaration of 1975, as revised in 2008.

\section{References}

Baker, L., Rideout, J., Gertler, P. and Raube, K. 2005: Effect of an internet-based system for doctor-patient communication on health care spending. Journal of the American Medical Informatics Association 12, 530-36.

Bishop, T.F., Press, M.J., Mendelsohn, J.L. and Casalino, L.P. 2013: Electronic communication improves access, but barriers to its widespread adoption remain. Health Affairs (Project Hope) 32, 1361-367.

Couchman, G.R., Forjuoh, S.N. and Rascoe, T.G. 2001: E-mail communications in family practice: what do patients expect? The Journal of Family Practice 50, 414-18.

Crotty, B.H., Tamrat, Y., Mostaghimi, A., Safran, C. and Landon, B.E. 2014: Patient-to-physician messaging: volume nearly tripled as more patients joined system, but per capita rate plateaued. Health Affairs (Project Hope) 33, 1817-822.

de Jong, C.C., Ros, W.J. and Schrijvers, G. 2014: The effects on health behavior and health outcomes of internet-based asynchronous communication between health providers and patients with a chronic condition: a systematic review. Journal of Medical Internet Research 16, e19.

Garrido, T., Meng, D., Wang, J.J., Palen, T.E. and Kanter, M.H. 2014: Secure e-mailing between physicians and patients: transformational change in ambulatory care. The Journal of Ambulatory Care Management 37, 211-18.

Hobbs, J., Wald, J., Jagannath, Y.S., Kittler, A., Pizziferri, L., Volk, L.A., Middleton, B. and Bates, D.W. 2003: Opportunities to enhance patient and physician e-mail contact. International Journal of Medical Informatics 70, 1-9.

Keplinger, L.E., Koopman, R.J., Mehr, D.R., Kruse, R.L., Wakefield, D.S., Wakefield, B.J. and Canfield, S.M. 2013: Patient portal implementation: resident and attending physician attitudes. Family Medicine 45, 335-40.

Kittler, A.F., Wald, J.S., Volk, L.A., Pizziferri, L., Jagannath, Y., Harris, C., Lippincott, M., Yu, T., Hobbs, J. and Bates, D.W. 2004: The role of primary care non-physician clinic staff in e-mail communication with patients. International Journal of Medical Informatics 73, 333-40.

Kummervold, P.E. and Johnsen, J.A. 2011: Physician response time when communicating with patients over the internet. Journal of Medical Internet Research 13, e79.

Lam, R., Lin, V.S., Senelick, W.S., Tran, H.P., Moore, A.A. and Koretz, B. 2013: Older adult consumers' attitudes and preferences on electronic patient-physician messaging.
The American Journal of Managed Care 19 (10, Spec No), eSP7-SP11.

Leong, S.L., Gingrich, D., Lewis, P.R., Mauger, D.T. and George, J.H. 2005: Enhancing doctor-patient communication using email: a pilot study. The Journal of the American Board of Family Practice 18, 180-88.

Liederman, E.M., Lee, J.C., Baquero, V.H. and Seites, P.G. 2005: The impact of patient-physician web messaging on provider productivity. Journal of Healthcare Information Management 19, 81-86.

Newhouse, N., Lupiáñez-Villanueva, F., Codagnone, C. and Atherton, H. 2015: Patient use of email for health care communication purposes across 14 European countries: an analysis of users according to demographic and health-related factors. Journal of Medical Internet Research $17, \mathrm{e} 58$.

Ontario Ministry of Health and Long-Term Care. 2013: 2012 Agreement for Ontario doctors. Retrieved 8 June 2016 from http://www.health.gov.on.ca/en/pro/programs/phys_services.

Patt, M.R., Houston, T.K., Jenckes, M.W., Sands, D.Z. and Ford, D.E. 2003: Doctors who are using e-mail with their patients: a qualitative exploration. Journal of Medical Internet Research 5, e9.

Plener, I., Hayward, A. and Saibil, F. 2014: E-mail communication in the management of gastroenterology patients: a review. Canadian Journal of Gastroenterology \& Hepatology 28, 161-65.

Santana, S., Lausen, B., Bujnowska-Fedak, M., Chronaki, C.E., Prokosch, H.U. and Wynn, R. 2011: Informed citizen and empowered citizen in health: results from an European survey. BMC Family Practice 12, 20.

Schickedanz, A., Huang, D., Lopez, A., Cheung, E., Lyles, C.R., Bodenheimer, T. and Sarkar, U. 2013: Access, interest, and attitudes toward electronic communication for health care among patients in the medical safety net. Journal of General Internal Medicine 28, 914-20.

Sibley, L.M. and Weiner, J.P. 2011: An evaluation of access to health care services along the rural-urban continuum in Canada. BMC Health Services Research 11, 20.

Singh, H., Fox, S.A., Petersen, N.J., Shethia, A. and Street, R.L. Jr. 2009: Older patients' enthusiasm to use electronic mail to communicate with their physicians: cross-sectional survey. Journal of Medical Internet Research 11, e18.

Statistics Canada. 2015: Chart 2.4. Proportion of population aged less than 15 years old and 65 years old and over, 1982 to 2036, Canada. Retrieved 14 June 2016 from http://www. statcan.gc.ca/pub/91-215-x/2012000/ct008-eng.htm.

Virji, A., Yarnall, K.S., Krause, K.M., Pollak, K.I., Scannell, M.A., Gradison, M. and Østbye, T. 2006: Use of email in a family practice setting: opportunities and challenges in patient- and physician-initiated communication. BMC Medicine 4, 18.

Ye, J., Rust, G., Fry-Johnson, Y. and Strothers, H. 2010: E-mail in patient-provider communication: a systematic review. Patient Education and Counseling 80, 266-73. 


\section{Feng Chang et al.}

\section{Appendix: Quantitative and qualitative surveys}

\section{Quantitative Client Survey}

Date Survey Completed _ (DD)/__ (MM) $/ 20$

1. How old are you? years

2. How would you rate your overall health?

Good

Fair

Poor

3. What is your gender?
Female
Male
Other

4. Which city/town do you live in?

5. What is your occupation?

6. What is the highest level of education you have completed?

$\square$ Less than high school

$\square$ Some college

$\square$ Skilled trades and apprenticeships

Professional degree (MD, JD, etc.)

$\square$ High school/GED

$\square$ Bachelor's degree/college degree

$\square$ Postgraduate degree holder

$\square$ Prefer not to answer

7. What is your household income?

Under $\$ 25,000 / \mathrm{yr}$

$\$ 50,000-\$ 74,999 / \mathrm{yr}$

$\$ 100,000 / y r$ or more

$\$ 25,000-\$ 49,999 / \mathrm{yr}$

$\$ 75,000-\$ 99,999 / \mathrm{yr}$

Prefer not to answer

8. Do you have access to the Internet at your home or place of residence?
$\square$ Yes
No
Not sure

9. Do you access the Internet from places other than your home (such as the library)?

$\square$ Yes. Where?

$\square$ No

Not sure

10. Do you have an e-mail account?

$\square$ Yes

11. How much time do you spend on the Internet?

$\square$ None
$\square$ 16-29 minutes
$\square$ 45-59 minutes
$\square 1$ hour 15 minutes - 2 hours

$\square$ 1-15 minutes
$\square$ 30-44 minutes
$\square 1$ hour -1 hour 14 minutes
$\square$ More than 2 hours

12. How much time do you spend on e-mail?

$\square$ None

16-29 minutes

1-15 minutes

30-44 minutes

1 hour -1 hour 14 minutes

45-59 minutes

More than 2 hours

Primary Health Care Research \& Development 2017; 18: 149-160 
13. Do you access any of these websites on a regular basis?

$\square$ Facebook (Other social networking websites)

$\square$ YouTube

$\square$ News/weather/sport websites

$\square$ E-mail websites (Gmail, Hotmail, Yahoo! Mail)

$\square$ Recreational use

$\square$ Wikipedia/other reference sources

$\square$ Others. Please list below in the blank lines

I don't access the Internet

Prefer not to answer

14. Have you ever used the Internet to learn about health or medical issues?

$\square$ Yes

$\square$ No

Not sure

I don't access the Internet

Prefer not to answer

15. Would you like to start using e-mail to communicate with your family doctor's office?

$\square$ Yes

I don't access e-mail

$\square$ No

$\square$ Prefer not to answer

16. How likely would you use e-mail to communicate with a family health team clinic staff member, if this service is available? (Please circle a number between 0 and 10, 0 being not very likely and 10 being most likely.)

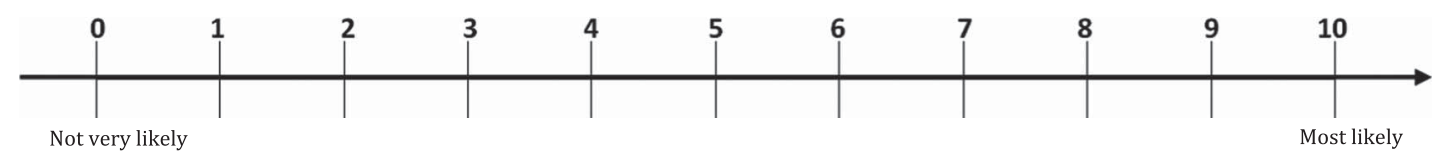

17. Which of the following e-mail services would be of interest to you? (check all that apply):

Asking questions about diet, medication, dressings and patient education resources

Disease monitoring for home glucose, blood pressure, weight or peak flow measurements, etc.

Administrative information such as referral requests, changes in contact information, work- or schoolrelated notes

Requests for prescription refills

Receiving laboratory test results and interpretation

Scheduling appointments

$\square$ Clarifications regarding issues discussed in person such as changes in your diet, medication and lifestyle, when to make follow-up appointments, etc.

Others. Please list.

Primary Health Care Research \& Development 2017; 18: 149-160 
158 Feng Chang et al.

\section{Qualitative Interview Guide - Clients}

Age Gender

Highest level of education

Occupation

Where do you live?
a. Listowel
b. Kitchener-Waterloo
c. Other community

1. In the last 12 months, how many times have you visited the clinic?

2. Who is your doctor?

3. Do you use the Internet in your daily life (personally/professionally)?

4. What do you use it for?

5. Do you use it for e-mail?

6. How much time do you spend on e-mail in your daily life?

7. Do you use e-mail for professional purposes?

8. Other than e-mail, what other sort of gadgets do you use in your daily life such as cell phone, smart phone, iPad, etc.?

9. For clients not having internet access: Would you be interested in getting Internet access if it let you communicate with the clinic staff?

10. For clients not having internet access: Would you be interested in learning how to e-mail if it let you communicate with the clinic staff?

11. On a scale from 0 to 10 , in general, how do you rate your satisfaction in communicating with the clinic ( 0 being not satisfied at all and 10 being very satisfied)

12. What would you improve in the clinic?

13. When you call the clinic, how long does it take to get an appropriate response? (to get an appointment, speak to the doctor, etc.)

14. How much time does it take for the clinic to get back to you on prescription refills and other questions?

15. What do you think e-mail can be used for in the clinic?

16. What are your thoughts on using e-mail to communicate with the clinic?

17. What problems do you see with using e-mail?

18. If you were able to e-mail staff, how long do you think they should take before they reply to you?

19. If you were able to e-mail staff, how long do you think they will take before they reply to you?

20. How comfortable do you think you will be using a new system like e-mail on a scale of 0 to 10 ( 0 being not ready at all and 10 being able to e-mail the clinic tomorrow)?

21. Do you think other clients will start using the service?

22. How do you think the new system will work for the clinic? 


\section{Qualitative Interview Guide - Healthcare Providers and Staff}

Intro

1. How many days are you at the clinic? Are these full days or half days? What is your regular weekly schedule?

2. How many clients do you have on your roster? (for MDs only)

3. Please describe your current practice.

Baseline personal e-mail

4. Do you use e-mail in your personal life?

5. What do you use it for?

6. How much time do you spend on personal e-mail in a typical day?

If don't use e-mail

7. Do you use the Internet? How much time do you spend on the Internet on a regular day?

8. What do you use it for?

Professional e-mail baseline

9. Do you use e-mail for work-related use? (Purkinje [EMR] messages don't count)

10. In your 8-hour workday, estimate how much time you spend on work-related e-mail.

11. Do you spend any time outside of these 8 working hours to catch up on work-related e-mails?

E-mail communication system

12. In relation to communicating with clients, what improvements would you make?

13. What role do you think e-mail has for communicating with clients?

14. What are your thoughts on using e-mail in the clinic?

Readiness for change

15. On a scale of 0 to 10 , how would you rate your readiness to use e-mail to communicate with clients? ( 0 being not ready at all and 10 being you could start using e-mail tomorrow)

16. On a scale of 0 to 10 , how would you rate your willingness to use e-mail to communicate with clients? ( 0 being not willing at all and 10 being you would be willing to start using e-mail tomorrow)

17. On a scale of 0 to 10 , how would you rate the clinic staff readiness such as additional training to use e-mail to communicate with clients? ( 0 being not ready at all and 10 being you could start using email tomorrow)

18. On a scale of 0 to 10 , how would you rate the readiness of clients to use e-mail to communicate with clinic staff? ( 0 being not ready at all and 10 being they could start using e-mail tomorrow)

Implementation

19. If the clinic were to start using e-mail to communicate with clients, do you think additional training would be required?

20. How do you think the system will be set up?

21. Who should be responsible for monitoring and responding to client e-mails?

22. What do you think client uptake will be?

23. What concerns, if any, do you have in using e-mail with clients?

24. What policies and guidelines are you aware of for e-mailing clients?

25. What polices and guidelines do you think would be necessary to set up in the clinic before e-mailing clients? 


\section{Qualitative Interview Guide - Pharmacists}

Intro

Age _ Note gender _ Pharmacy

Intro

1. How many days are you at the pharmacy? Are these full days or half days? What is your regular weekly schedule? How long is each shift?

2. Estimate how many clients you have that fill prescriptions here on a daily basis.

3. Estimate how many of those clients are from the NPFHT (Listowel Clinic). (Percentage, fraction or proportion)

Baseline personal e-mail

4. Do you use e-mail in your personal life?

5. What do you use it for?

6. How much time do you spend on personal e-mail in a typical day?

If don't use e-mail

7. Do you use the Internet? How much time do you spend on the Internet on a regular day?

8. What do you use it for?

Professional e-mail baseline

9. Do you use e-mail for work-related use?

10. In your 8-hour workday, estimate how much time you spend on work-related e-mail.

11. Do you spend any time outside these 8 working hours to catch up on work-related e-mails?

12. Do you have a work e-mail address? If not, does your store have an e-mail address?

13. Can you check this e-mail address? How often do you log into it?

Access to clinic staff

14. In a typical shift, how often and why do you contact the NPFHT?

15. How do you contact the NPFHT?

16. How much time does it take for the NPFHT to get back to you? For prescription refills? Answering questions on scripts?

17. What are your thoughts on using e-mail to communicate with the clinic?

Client and practice uses

18. What problems do you see with using e-mail?

19. On a scale of 0 to 10 , how would you rate the readiness of clients to use e-mail to communicate with clinic staff? ( 0 being not ready at all and 10 being they could start using e-mail tomorrow)

20. What do you think client uptake will be?

21. In the pharmacy, who will be responsible for sending out and receiving those e-mails?

22. What policies do you think will have to be in place before you can start e-mailing the clinic?

23. How do you think the new system will work for the pharmacy?

24. What other uses do you see e-mail having in current pharmacy practice? 